\title{
Emergent spatial structures in critical sandpiles
}

\author{
Bosiljka Tadić ${ }^{1, *}$ and Deepak Dhar ${ }^{2, * *}$ \\ ${ }^{1}$ Jožef Stefan Institute, P.O. Box 3000, 1001 Ljubljana, Slovenia \\ 2 Theoretical Physics Group, Tata Institute of Fundamental Research, Homi Bhabha Road, Mumbai 400005, India
}

\begin{abstract}
We introduce and study a new directed sandpile model with threshold dynamics and stochastic toppling rules. We show that particle conservation law and the directed percolation-like local evolution of avalanches lead to the formation of a spatial structure in the steady state, with the density developing a power law tail away from the top. We determine the scaling exponents characterizing the avalanche distributions in terms of the critical exponents of directed percolation in all dimensions.
\end{abstract}

Many extended slowly driven dissipative systems in nature evolve into self-organized critical (SOC) steady states which show long-range spatial and temporal correlations. Since the pioneering work of Bak et al. [1], sandpile models have served as paradigms of SOC systems. A great deal of understanding of SOC has been achieved by numerically studying sandpile models with different evolution rules [2]. For a special subclass of these models, i.e., the Abelian Sandpile Models (ASM), several analytical results are available [3]. Recent studies of models with stochastic toppling rules have shown that these models usually belong to a universality class different from deterministic automata; they have robust critical states with respect to changes of a control parameter, and may also exhibit a dynamical phase transition between qualitatively different steady states [4]. However, the spatial structures in the steady states of these models are much less investigated [5]. Due to long-range correlations in the critical states, influence of the boundary can be felt deep inside, and this can give rise to large-scale spatial structures. Indeed, emergent spatial structures are sine qua non for a SOC theory of fractals occurring in nature, e.g., mountain landscapes, river networks, and earthquake fault zones.

In this Letter, we propose a new stochastic sandpile model which shows emergent spatial structures in the steady states. The model is a stochastic generalization of the directed ASM [6] and contains a probabilistic control parameter $p$. In the case $p=1$ the exponents are known exactly in all dimensions [6]. We show that for $p \neq 1$, the model is in a new universality class and can be related to directed percolation (DP) [7] problem with a nonuniform concentration profile. A steady state exists only for $p>p^{\star}$, where $p^{\star}$ equals the critical threshold for directed site percolation. Above $p^{\star}$ the system evolves towards a steady state which is arbitrarily close to the generalized DP critical line. We also show that in the critical state of our model a spatial structure results from an interplay of DP-like local evolution rules on the one side, and the dynamic conservation law on the other. We find a power-law density profile, which further enables us to determine the exact expressions for the scaling exponents of avalanches in terms of the DP critical exponents in all dimensions $d$. Our numerical simulations in two dimensions support these conclusions.

For concreteness, we consider a square lattice of linear size $L$ with sites $(i, j)$ oriented so that the diagonal direction $(1,1)$ is vertically down. A non-negative integer variable, height $h(i, j)$, is attached to each lattice site. Sand grains are added one at a time at a randomly chosen site on the top layer, increasing its height by one. A site becomes unstable if $h(i, j) \geq 2$ and relaxes as follows: With probability $p$, the local height decreases by two, and heights at each of its two downward neighbors increase by one. Otherwise, the heights remain unchanged. In either case, the site is considered as stable at the next time step. Only sites to which at least one particle was added at the preceding time step are checked for toppling. A discrete-time parallel update is applied to all such sites. We apply periodic boundary conditions in the horizontal direction. Two particles leave the system for each toppling occurring at the bottom layer.

In the limit $p=1$, the structure of the steady state is known exactly [6]: Only configurations with heights $h=0$ and $h=1$ occur, and all such configurations are equally probable. The avalanche clusters are compact. For stochastic toppling, $p \neq 1$, the model has quite a different behavior. It is no longer Abelian, since adding two particles together to a site of height $h \geq 2$ does not have the same effect as adding them one by one at different time steps. (In the latter case, toppling can occur twice with a finite probability.) With a small probability heights could become arbitrarily large. The avalanche clusters have branches and holes of various sizes. An example is shown in Fig. 1.

For small $p$ the average influx of particles per attempted toppling at a site, which is $\geq 1$, exceeds the average outflux, $2 p$, thus there exists a value $p^{\star}$, such that a steady state is possible only for $p \geq p^{\star}$. We now argue that $p^{\star}=p_{c}$, where $p_{c}$ is the critical threshold for 
the directed site percolation problem [8].

Suppose that $\mathcal{C}$ is a stable configuration of the pile, and a particle added at the site $(i, j)$ causes on the average $n_{\ell}(\mathcal{C})$ topplings at depth $\ell$ below it. If for any stable configuration $\mathcal{C}^{\prime}$, all heights are greater than or equal to corresponding heights in $\mathcal{C}$ then $n_{\ell}\left(\mathcal{C}^{\prime}\right) \geq n_{\ell}(\mathcal{C})$. Now, note that for all configurations $\mathcal{C}$ for which all sites have $h \geq 1$, the distribution of size of avalanches is exactly the same as in the directed site-percolation problem on this lattice. Therefore, for all $p<p_{c}, n_{\ell}$ decreases exponentially with $\ell$. Hence no topplings occur at large depths, and particles pile up in the upper layers, and thus there is no steady state. Conversely, for $p>p_{c}$, the directed percolation avalanche clusters typically form a wedge, and $n_{\ell}$ increases with $\ell$ for large $\ell$ (see below). Then avalanches in configurations with all heights $h \geq 1$ cause many topplings, and each layer after the avalanche on the average loses particles. Thus if the system ever reaches a state with large density, the number of particles in the system will decrease until at sufficiently many sites heights become low enough so that the propagation of avalanches is affected, and it becomes critical, but not super-critical. Hence the system will have a steady state for all $p \geq p^{\star}=p_{c}$. On the square lattice numerical estimate for $p_{c}$ [7] gives $p^{\star} \approx 0.7054853(5)$.

Our numerical simulations support this conclusion. In Fig. 2 the probability $P(T)$ that an avalanche has durations $\geq T$ is plotted against $T$ for different values of $p$ and $L=200$. (Notice that in directed models $T \equiv \ell$ ). For large $T$ it varies as $P(T) \sim T^{1-\tau_{t}}$. Exponential decay of the lower three curves indicates loss of SOC. In the steady states for $p \geq p^{\star}$ the integrated cluster-size distribution behaves as $D(s) \sim s^{1-\tau_{s}}$, with the following scaling properties

$$
D(s, L)=L^{1-\tau_{t}} \mathcal{D}\left(s L^{-D_{\|}}\right),
$$

and the scaling relation $\left(\tau_{s}-1\right) D_{\|}=\tau_{t}-1$ is satisfied. In Fig. 3 the distribution $D(s, L)$ vs. $s$ and its finite-sizescaling plot are shown for $p=p^{\star}$.

We now discuss the structure of the steady state for $p^{\star} \leq p<1$. Let $\rho$ be the probability that a site chosen at random has a nonzero height in the steady state. Then the probability that this site will topple if a single particle is added to it is $P_{1}=p \rho$, and the probability that it topples if two particles are added to it is $P_{2}=p$. The correlations of heights on the same layer are irrelevant and can be ignored [9]. Therefore, the evolution of an avalanche is the same as in a Domany-Kinzel (DK) cellular automaton model of directed site-bond percolation [10]. This implies that, in order for the system to have critical correlations in the bulk, the set of points $\left(P_{1}, P_{2}\right)$ must lie on the critical line of the DK model.

However, as we show below, the dynamic conservation law prevents the avalanche clusters in the steady state from being in the universality class of DP. Particle conservation implies that in the steady state the average number of topplings at each layer equals $1 / 2$. On the other hand, in the case of DP the expected number of growth sites at depth $\ell$ is known to vary as $m \sim \ell^{\kappa}$ with $\kappa=\left[(d-1) \nu_{\perp}-2 \beta\right] / \nu_{\|}$, where $\beta, \nu_{\|}$and $\nu_{\perp}$ are standard DP critical exponents of the order parameter, and parallel and transverse correlation lengths, respectively. For DP in $d<5$ dimensions $\kappa>0$, thus $m$ increases with $\ell$, which is clearly not possible in the steady state. The way these conflicting requirements of particle conservation and locally critical DP-like evolution are satisfied in our model is that the critical steady state develops a spatial structure. The density $\rho$, and hence $P_{1}$, are not uniform throughout the system, but vary from layer to layer [11]. Let $\rho(\ell)$ be the average density of sites with non-zero height in the $\ell$-th layer. By equating average influx and outflux of particles at a site on $\ell$-th layer, we find that $\rho(\ell)=[1-(2 p-1) f(\ell)] /[2 p(1-f(\ell)]$, where $f(\ell)$ is the number of topplings caused by simultaneous addition of two particles at the site. The exactly calculated values of $f(\ell)$ for the first few layers indicate that $\rho(\ell)$ increases with $\ell$. As discussed above, for large $\ell$ the profile reaches the value $\rho^{\star}(p)=P_{1}^{\star}(p) / p$, where $\left(P_{1}^{\star}(p), p\right)$ is a point on the DP critical line in the $\left(P_{1}, P_{2}\right)$ parameter space. In Fig. 4 , we plot the profile $\rho$ against $\ell$ obtained by numerical simulations for $p=p^{\star}$ and $L=200$. The profile is well described by a power law:

$$
\rho(\ell)=\rho^{\star}(p)-A(p) \ell^{-x},
$$

with $\rho^{\star}=1$ and $A=0.39$ for $p=p^{\star}$, and $x=0.578$.

We now show that the profile given by Eq. (21) changes the avalanche statistics in our model, and thus strongly affects the bulk transport. In the bulk, transport of particles is locally described by the DK model with parameters $\left(P_{1}(\ell), P_{2}\right)$. For large $\ell$ the system is close to the critical line and the local longitudinal correlation length $\xi(\ell)$ varies as $\xi(\ell) \sim\left[P_{1}^{\star}-P_{1}(\ell)\right]^{-\nu_{\|}}$. In order for the transport to propagate further to a distance $\ell, \xi(\ell)$ must be proportional to $\ell$, i.e.,

$$
\xi(\ell) \approx \ell / B
$$

This implies that the exponent $x$ in Eq. (2) is exactly $x=1 / \nu_{\|}$. From the simulation data in $\log (1-\rho(\ell))$ vs. $\log \ell$ plot we find the slope $x=0.575 \pm 0.005$ (see inset to Fig. 4), leading to $\nu_{\|}=1.738 \pm 0.005$, in a good agreement with $\nu_{\|}$for DP in two-dimensions [7].

The calculation of avalanche exponents for our model reduces to the problem of determining the distribution of cluster-sizes of surface clusters in a directed site-bond percolation model where the concentration of bonds has a power-law profile (2). In the renormalization-group approach [12] $x=1 / \nu_{\|}$is a marginal case and the cluster exponents may depend on the amplitude $A$.

Let $G\left(R_{\perp}, R_{\|}\right)$be the probability that the site $\left(R_{\perp}, R_{\|}\right)$topples if a particle is added at $(0,0)$ in the 
steady state. Since $\rho^{\star}(p)-\rho(\ell, p) \ll 1$ for large $\ell$, we can show that to leading order of perturbation

$$
G\left(R_{\perp}, R_{\|}\right) \approx G_{0}\left(R_{\perp}, R_{\|}\right) \exp \left[-\int_{1}^{R_{\|}} d \ell / \xi(\ell)\right],
$$

where $G_{0}\left(R_{\perp}, R_{\|}\right)$is the two-point correlation function for the critical DP process. Using Eq. (3) we get

$$
G\left(R_{\perp}, R_{\|}\right)=G_{0}\left(R_{\perp}, R_{\|}\right) R_{\|}^{-B} .
$$

The value of $B$ selected by the steady state is determined by the requirement that the average outflux of particles per avalanche from the $R_{\|}$-th layer equals one, i.e., $\sum_{R_{\perp}} G\left(R_{\perp}, R_{\|}\right) \sim 1$. Since in DP the average outflux is $\sum_{R_{\perp}} G_{0}\left(R_{\perp}, R_{\|}\right) \sim R_{\|}^{\left[(d-1) \nu_{\perp}-2 \beta\right] / \nu_{\|}}$, it follows that

$$
B=\left[(d-1) \nu_{\perp}-2 \beta\right] / \nu_{\|},
$$

where $\beta, \nu_{\|}$and $\nu_{\perp}$ are as above the DP exponents. Thus both the power-law tail and the amplitude $B$ are expressed in terms of standard DP exponents. These facts, in turn, determine the statistics of avalanche clusters. In addition to the exponents for the distributions of duration, $\tau_{t}$, and size, $\tau_{s}$, we also define the anisotropy exponent $\zeta$ for the average transverse extent $R_{\perp} \sim \ell^{\zeta}$ of a cluster of length $\ell$. Near the DP critical line $R_{\perp}$ is expected to have the scaling behavior as

$$
R_{\perp} \sim \ell^{\zeta_{D P}} \phi\left(\left[P_{1}^{\star}-P_{1}(\ell)\right] \ell^{1 / \nu_{\|}}\right)
$$

Notice that due to the power-law profile (2), the argument of the scaling function $\phi$ in Eq. (7) remains finite in the limit $\ell \rightarrow \infty$, and thus $\zeta=\zeta_{D P}=\nu_{\perp} / \nu_{\|}$.

In the critical DP, the probability that a perturbation survives up to layer $T$ varies as $P_{0}(T) \sim T^{-\beta / \nu_{\|}}$. As an expression similar to Eq. (4) applies also to the survival probability in the steady state of our model, we have that $P(T)=P_{0}(T) T^{-B}$. Using $B$ from Eq. (6) this gives

$$
\tau_{t}=1+(d-1) \zeta-\beta / \nu_{\|} .
$$

Using standard scaling arguments for the directed SOC system [6] we notice first that the expected number of topplings in a cluster of length $\ell$ scales as $\langle s\rangle_{\ell} \sim \ell^{\tau_{t}}$, that is, $D_{\|}=\tau_{t}$. Together with $\left(\tau_{s}-1\right) D_{\|}=\tau_{t}-1$ this then gives the renormalized $\tau_{s}$ exponent as

$$
\tau_{s}=2-1 / \tau_{t}
$$

Inserting the best known numerical values of the exponents for two-dimensional DP $[7]$, gives $\tau_{t}=1.47244, \tau_{s}$ $=1.32059$, and $\zeta=0.63261$. We have checked these predictions against numerical simulations of the exponents and fractal dimension $D_{\|}$. In the inset to Fig. 2 various scaling exponents are plotted versus $p$ in the scaling region $p \geq p^{\star}$. Away from a small crossover region near the point $p=1$, the obtained values of the exponents are independent of $p$ within numerical error. We find $\tau_{t}=1.460$ $\pm 0.014, \tau_{s}=1.313 \pm 0.012$, and $\zeta=0.624 \pm 0.014$ in fair agreement with the above conclusions.

For $d=3$ using Eqs. (85) and known numerical values of DP exponents [13] we find $\tau_{t}=1.674$ and $\tau_{s}=1.403$. The upper critical dimension of our stochastic model is $d_{c}=5$, in contrast to $d_{c}=3$ in the deterministic limit $p=1$. For $d \geq 5$, the DP critical exponents are $\beta=1$, $\nu_{\|}=1$, and $\nu_{\perp}=1 / 2$, leading to $B=0$, and thus the exponents have the mean-field values $\tau_{t}=2$, and $\tau_{s}=3 / 2$.

In conclusion, we have demonstrated that nearness of the steady states to the directed-percolation critical line and the conservation of number of particles in the bulk are responsible for the emergent spatial structures in our stochastic sandpile model. A power-law density profile has been found and the self-organized criticality which is in a different universality class from the deterministic limit. In all dimensions $d$ the scaling exponents of avalanches have been determined in terms of standard directed percolation critical exponents.

The work of B.T. was supported by the Ministry of Science and Technology of the Republic of Slovenia. B.T. thanks Al Corral for his assistance in the graphic program. D.D. would like to acknowledge useful comments on the manuscript by M. Barma and S.N. Majumdar.

* Electronic address: Bosiljka.Tadic@ijs.si

** Electronic address: ddhar@theory.tifr.res.in

$1 \quad$ P. Bak, C. Tang and K. Wiesenfeld, Phys. Rev. Lett. 59, 381 (1987); Phys. Rev. A 38, 364 (1988).

2 L. P. Kadanoff, S. R. Nagel, L. Wu and S. M. Zhou, Phys. Rev. A 39, 6524 (1989); S. S. Manna, Physica A 179, 249 (1991); A. B. Chabra, M. J. Feigenbaum, L. P. Kadanoff, A. J. Kolan, and I. Procaccia, Phys Rev E 47, 3099 (1993); B. Tadić and R. Ramaswamy, Phys. Rev. E 54, 3157 (1996).

3 D. Dhar, Phys. Rev. Lett. 641613 (1990); S. N. Majumdar and D. Dhar, Physica A 185, 129 (1992); D. Dhar and S. S. Manna, Phys. Rev. E 49, 2684 (1994).

4 Y.-C. Zhang, Phys. Rev. Lett. 63, 470 (1989); S.S. Manna, J. Phys. A 24, L363 (1992); S. Maslov and Y.C. Zhang, Physica A 223, 1 (1996); A. Ben-Hur and O. Biham, Phys. Rev. E 53, R1317 (1996); S. Lübeck, B. Tadić, and K.D. Usadel, Phys. Rev. E 53, 2182 (1996).

5 A. A. Ali, Phys. Rev. E 52, R4595 (1995); S. Lübeck, K.D. Usadel, and B. Tadić, in Fractal Reviews in the Natural and Applied Sciences, Ed. M.M. Novak, p. 47, Chapman and Hall (London) 1995.

6 D. Dhar and R. Ramaswamy, Phys. Rev. Lett. 63, 1659 (1989).

7 I. Jensen, J. Phys. A 29, 7013 (1996) and refs. therein.

8 See also M. Markošová, M. H. Jensen, K.B. Lauritsen, 
and K. Sneppen, Phys. Rev. E55, R2085 (1997).

9 The argument becomes exact with the rules modified such that heights at sites on the same layer are randomly redistributed fter each avalanche.

10 E. Domany and W. Kinzel, Phys. Rev. Lett. 53, 311 (1984).

11 J. G. Brankov, E. V. Ivashkevich and V. B. Priezzhev, J Phys (Paris) I 3, 1729 (1993) and E. V. Ivashkevich, J. Phys. A 27, 3643 (1994) found a density profile in the simpler ASM, with no significant effects on the critical state in the bulk.

12 For a review see F. Igloi, I. Peschel and L. Turban, Adv. Phys. 42, 685 (1993).

13 P. Grassberger, J. Phys. A: Math. Gen. 22, 3673 (1989).

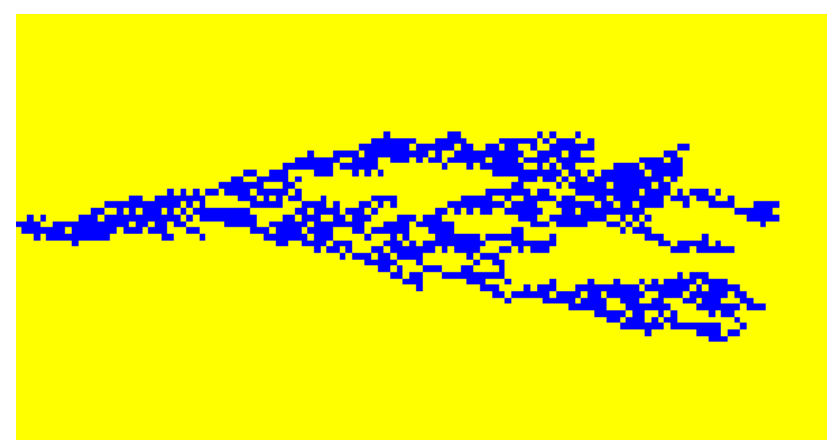

FIG. 1. Example of an avalanche running from left to right (dark) for $p=p^{\star}$ on the lattice of linear size $L=128$.

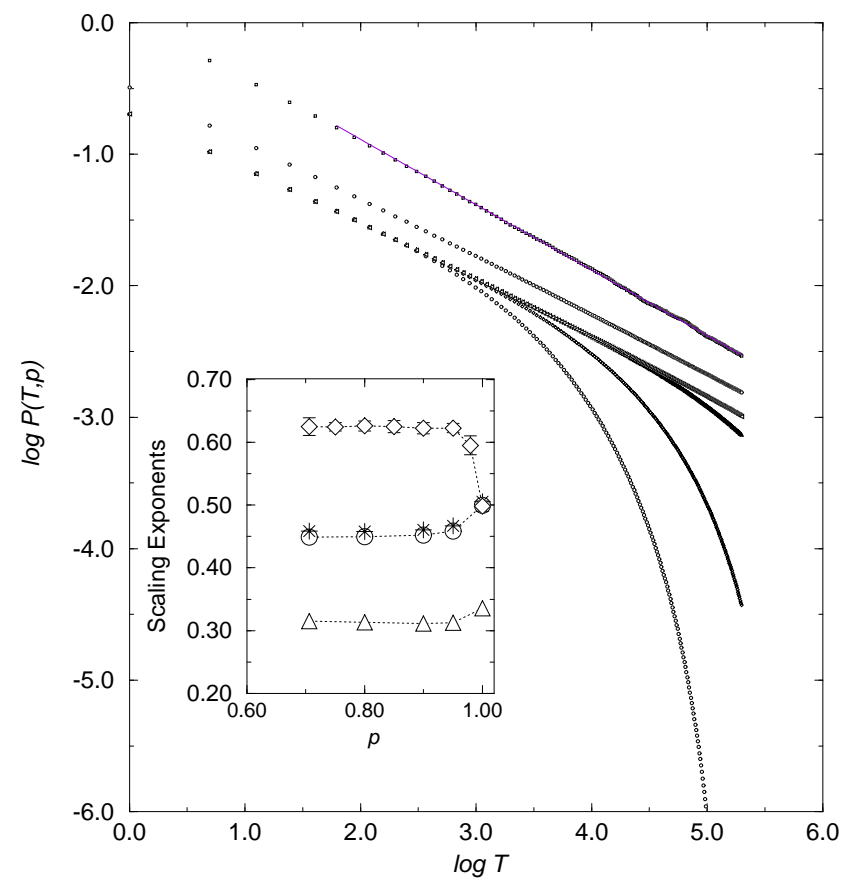

FIG. 2. Double logarithmic plot of the integrated distribution of durations $P(T, p)$ vs. $T$ for $L=200$, and $p=1,0.8$, $0.70548,0.69,0.68$, and 0.65 (top to bottom). First two curves have been shifted vertically for easier display. Inset: Scaling exponents: $(\diamond) \zeta,(\bigcirc) \tau_{t}-1,(\triangle) \tau_{s}-1$, and $(\star) D_{\|}\left(\tau_{s}-1\right)$ plotted against $p$ in the scaling region.

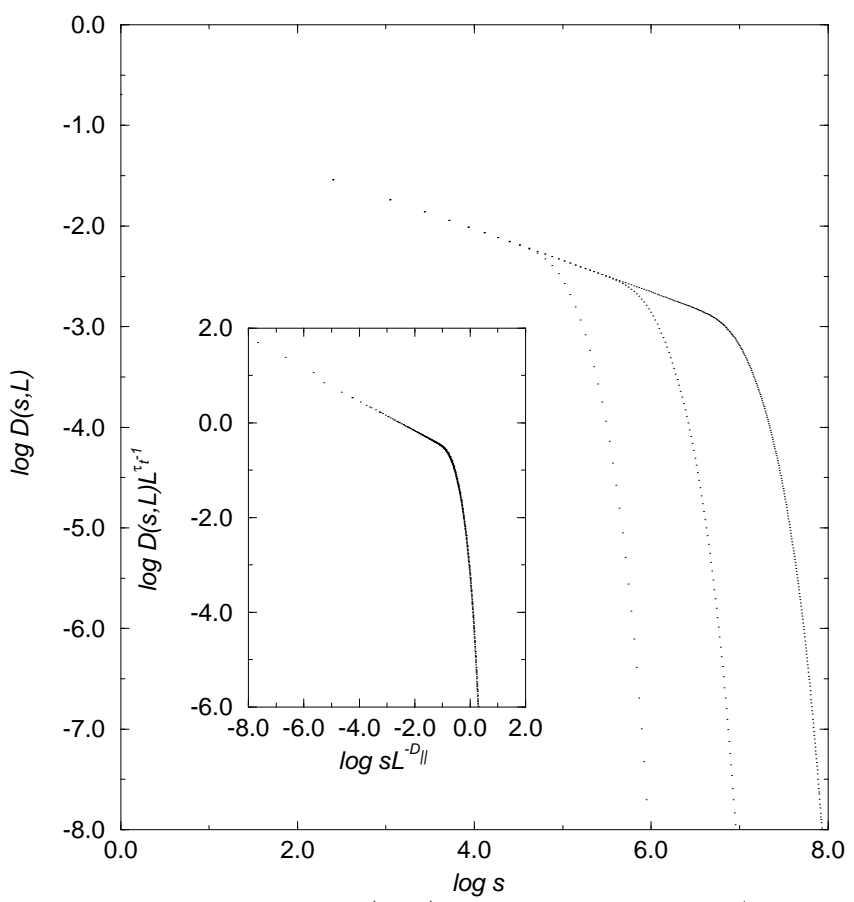

FIG. 3. Plot of $\log D(s, L)$ vs. $\log s$ for $p=p^{\star}$ and for three different lattice sizes $L=50,100$, and 200 . Inset: Data collapse according to Eq. (1), where we used the values $\tau_{t}-1=0.45$ and $D_{\|}=1.45$.

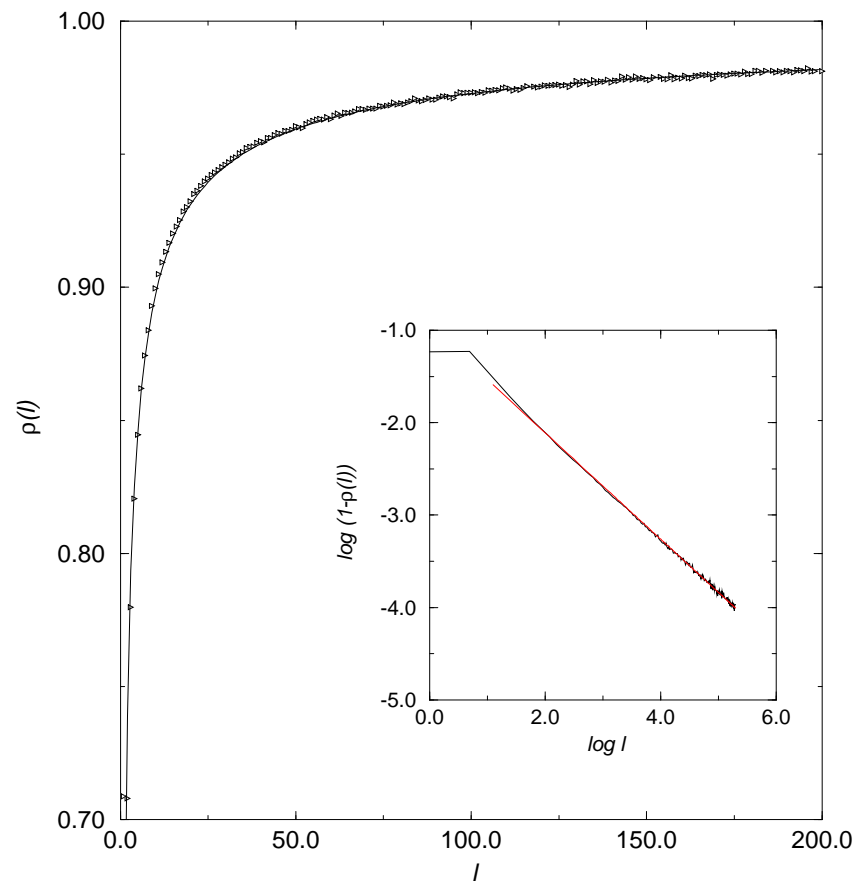

FIG. 4. Density profile $\rho(\ell)$ for $p=p^{\star}$ plotted against the distance $\ell$ from the top (triangles) and the theoretical curve $\rho(\ell)=1-0.39 \ell^{-0.578}$ (full line). Inset: Data in double-logarithmic plot. The slope is $x=0.575 \pm 0.005$. Within numerical error $x$ remains $p$-independent in the region $p \geq p^{\star}$. 